\title{
Optimal Allocation of Synchrophasor Units in the Distribution Network Considering Maximum Redundancy
}

\author{
Subhashree Priyadarshini \\ School of Electrical Engineering \\ Kalinga Institute of Industrial Technology \\ Deemed to be University \\ Bhubaneswar, India \\ subhashre9@rediffmail.com
}

\author{
Chinmoy Kumar Panigrahi \\ School of Electrical Engineering \\ Kalinga Institute of Industrial Technology \\ Deemed to be University \\ Bhubaneswar, India \\ panigrahichinmoy@gmail.com
}

\begin{abstract}
Phasor Measurement Unit (PMU) is a smart measuring device commonly used in wide-area monitoring systems. It provides the synchronized phasor values and the magnitudes of voltages and currents in real-time for the proper state calculation of the electrical network in a common time reference frame. But in order to avoid unnecessary placements, minimize installation cost, and due to the lack of communication facilities at the substations, the placing of PMUs at every location is not possible. Therefore several optimization techniques have been developed to solve the Optimal PMU Placement (OPP) problem. The OPP problem aims to reduce the number of PMUs by achieving a completely observable network. Many solutions to the OPP problem have been proposed for the transmission networks with the use of conventional and heuristic-based approaches, but very few for distribution networks. In this paper, the Binary Grey Wolf Optimization (BGWO) algorithm is proposed to solve the OPP problem considering the measurement redundancy (MR) to achieve complete observability of the distribution network. Finally, case studies have been done by implementing the proposed algorithm on different IEEE test feeders such as the IEEE $-13,-33,-37$, and -123 node feeder systems. The obtained results are compared with previous studies to verify the feasibility and efficiency of the proposed technique.
\end{abstract}

Keywords-binary grey wolf optimization; measurement redundancy; observability; optimal PMU placement; phasor measurement unit

\section{INTRODUCTION}

Proper monitoring, control, and protection of a power system can only be possible with complete observability of the entire network. A completely observable system provides accurate control and protection schemes for the secure and reliable operation of the electrical network. The invention of the Synchrophasor Unit or Phasor Measurement Unit (PMU) has made it possible by providing real-time measurements of the voltage and the current signals $[1,2]$. The measurements obtained from PMUs are accurate and time-stamped as they are connected with the Global Positioning System (GPS). The installation of PMU at different bus locations monitors the whole network by providing an accurate state estimation of each bus, but it is not possible to place PMUs at each bus as the installation includes the high cost of PMU devices along with its communication infrastructure and the data handling capacity. This leads to the optimal PMU placement problem. Therefore, the basic objective of the OPP problem is to optimize the PMU numbers to a minimum value by achieving the complete observability criterion. Many researchers have solved this OPP problem by using both traditional as well as heuristic optimization algorithms. Heuristic methods are capable of solving the placement problem in small and large networks with less computational time as compared to the traditional methods.

The most common traditional approach is the Integer Linear Programming (ILP). A Binary ILP (BILP) method is implemented in [3] to find minimum PMUs for the OPP problem in the distribution network considering the topology changes. Similarly, the BILP is implemented in [4] to solve the placement problem of PMUs with the consideration of communication links for the distributed state estimation in the distribution network. A hybrid ILP-based method is proposed in [5] to solve the OPP problem for the distribution network by finding all the possible solutions with observability. A heuristic Binary-based Particle Swarm Optimization (BPSO) algorithm is proposed in [6] to minimize the PMU number and maximize redundancy. The proposed method was implemented in a transmission network. Another improved BPSO has been proposed in [7] to find minimum PMUs with complete observability. The observability analysis was done by the state estimation of each bus in the network. Authors in [8] developed a new Genetic Algorithm (GA) to solve the PMU placement issue to achieve a complete observable and secure system. A non-dominated sorting GA was proposed to place PMUs optimally as well as to maximize the redundancy for IEEE 39 and 118 test bus transmission network in [9]. Ant colony optimization was implemented in [10] to minimize the PMUs by achieving maximum redundancy under normal and contingency conditions. An Artificial Bee Colony optimization (ABC) algorithm was implemented in [11] to find minimum PMUs in the presence of conventional flow measurements and zero injection buses. A multi-objective problem was solved for OPP using a binary-based Gravitational Search Algorithm (GSA) in [12]. The binary Jaya optimization was implemented

Corresponding author: Subhashree Priyadarshini 
to minimize the PMU numbers with complete network observability in [13]. This approach was only implemented in IEEE standard transmission networks. The Recursive Tabu Search (RTS) method was proposed to reduce the PMU devices by using numerical based observability analysis in [14]. The proposed method was only tested on the IEEE test bus transmission networks. Teaching-learning based optimization has been suggested in [15] to find the minimum PMUs required for the monitoring of the transmission network.

All these traditional and heuristic approaches have been implemented in transmission networks. Only the traditional approaches were used for solving the OPP in the distribution network. In this paper, a binary-based grey wolf optimization technique is developed to minimize the PMUs with complete observability and to maximize the measurement redundancy for distribution networks.

\section{PROBLEM FORMULATION}

The OPP problem is defined as the minimization of the number of PMUs required for monitoring the electrical network. In this paper, the purpose of solving the OPP problem is to reduce the number of PMUs in the distribution system. As a result, the overall installation cost can be minimized. The concept of maximum measurement redundancy is also considered in the OPP problem with complete observability in the objective function. Hence, the objective function $J(z)$ for the distribution network can be expressed as [16]:

$$
\begin{array}{r}
\min J(z)=\sum_{i=1}^{N_{D}} z_{i}+w_{1}\left(M-A^{\prime} Z\right)^{T}\left(M-A^{\prime} Z\right)+ \\
\\
w_{2}\left(N_{o b}\right) \text { subject to } A^{\prime} \cdot Z \geq b
\end{array}
$$

where $N_{D}$ is the total number of nodes in the feeder, $w_{1}$ and $w_{2}$ are the weight parameters, $N_{o b}$ is the total number of observable nodes in the system, and $z_{i}$ is the element of binary decision vector $(Z)$ which denotes the PMU placement at the $i$ th node and it can be defined as follows:

$$
z_{i}=\left\{\begin{array}{c}
1, \text { if } P M U \text { is placed at node } i \\
0, \text { otherwise }
\end{array}\right.
$$

$A^{\prime}$ is the binary connectivity matrix derived from the connection of each node in a distribution network.

$$
\begin{aligned}
& \left(A^{\prime}\right)_{n \times n}(i, j)=\left\{\begin{array}{c}
1, \text { if node } i=\text { node } j \\
1, \text { if node } i \text { is connected to } j \\
0, \text { otherwise }
\end{array}\right. \\
& b_{n \times 1}=\left[\begin{array}{lllllll}
1 & 1 & 1 & \ldots & \ldots & \ldots
\end{array}\right]^{T}=\text { unit vector } \\
& M=\left[\begin{array}{lllll}
M_{1} M_{2} M_{3} & \ldots \ldots & \ldots & M_{N_{D}}
\end{array}\right]_{1 \times N_{D}}^{T}
\end{aligned}
$$

The entries of each element in the $M$ vector are defined as the connectivity of each node plus one. For example, node 2 in Figure 1 is connected with 3 branches. Therefore the maximum value of node 2 is 4 \{ 3 for the connected branches (node 1, node 3 , and node 4 ) and 1 for the node itself $\}$. Hence the values of each element are set to be more than the actual connections. In (5), the minimization of the difference between the desired value $(M)$ and the actual value (obtained from PMU placement) is nothing but the maximization of measurement redundancy. The placement set with a minimum MR value will give the best placement result.

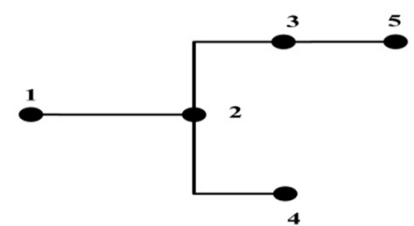

Fig. 1. Basic diagram of a 5 node feeder network.

A completely observable electrical network is defined when all the nodes are directly or indirectly monitored by the installed PMUs [17]. Therefore, the PMU measurement method is divided into two categories. The first one is the direct measurement method and the second one is the indirect measurement method. The node at which a PMU is placed can directly monitor that node and also the connected nodes to it. Hence, it is known as the direct measurement method. In indirect measurement, Kirchhoff's law is used to calculate the unknown current value and the voltage value of the nodes. Based upon these two laws, observability rules are developed and expressed as:

Rule 1: A node with a known voltage value along with its current value gives the unknown voltage value of the other end node.

Rule 2: If the voltage values of two connected nodes are known, then the current that flows between them can be calculated by using Ohm's law and KCL.

In this work, the solution for the placement problem is solved by considering the normal operating condition and single PMU loss.

\section{A. Single PMU Loss}

Single PMU loss is an important contingency during which the monitoring of the entire network can be affected. Therefore, to avoid such problems in the distribution system, each node should be observed by at least two PMUs for the continuation of proper monitoring and control of the entire network [18]. Hence the unit vector in (4) can be modified as:

$$
b_{n \times 1}=\left[\begin{array}{llllll}
2 & 2 & 2 & \ldots & \ldots \ldots
\end{array}\right]^{T}
$$

\section{B. Measurement Redundancy (MR)}

The measurement redundancy calculation is necessary for every OPP solution set for the secure and quality operation of the electrical network. Since the proposed BGWO technique is a meta-heuristic based approach, multiple placement sets will be obtained with an equal number of minimum PMUs to achieve a completely observable system. Therefore, the calculation of the Bus Observability Index (BOI) and the Complete System Observability Index (CSOI) are required to maximize the redundancy [19]. BOI represents the total number of times each node is observed from the OPP solution set, whereas CSOI is the summation of all the BOIs calculated for each node in the distribution network. The highest CSOI value yields a better placement location for the PMU installation. In this paper, the MR concept is considered to evaluate and compare the best placement sets obtained from the proposed and previously developed techniques. The expressions for BOI and CSOI are given in (7) and (8): 


$$
\begin{gathered}
\mathrm{BOI}_{i}=A^{\prime} \cdot Z_{i} \\
C S O I=\sum_{i=1}^{N_{D}} B O I_{i}
\end{gathered}
$$

\section{BINARY GREY WOLF TECHNIQUE}

Grey Wolf Optimization (GWO) is a newly developed population-based meta-heuristic technique influenced by the organizational structure and hunting nature of grey wolves [20]. Here, the binary-based GWO technique is proposed to solve the OPP problem along with maximum redundancy for the distribution system. The grey wolf family mainly consists of four different kinds of members, starting with the head of the group commonly known as the alpha wolf $(\alpha)$, which is the leader of the family and responsible for the decision making. The next member is known as the beta wolf $(\beta)$. It follows the order of the alpha wolf and commands the other wolves. The third kind is known as the delta wolf $(\delta)$ which follows the commands given by the alpha and beta wolves. The last member of the group is the omega wolf $(\omega)$. The omega wolves are dominated by all the above three types of wolves. The hunting activity of grey wolves follows three basic steps. The first step is the tracking of the desired prey, the next step is the encircling of the prey, and the last step is harassing and attacking the prey.

Mathematically, the hunting process of the prey starts with the alpha $(\alpha)$, beta $(\beta)$, and delta $(\delta)$ wolves, while the omega $(\omega)$ wolves only follow the instructions provided by the three leaders and update their positions according to them. The following mathematical equations describe the encircling behavior of the grey wolves [20].

$$
\begin{aligned}
& D=\left|C\left(X_{P}(t)\right)-X(t)\right| \\
& \text { and } X(t+1)=\left\{X_{P}(t)-A \cdot D\right\}
\end{aligned}
$$

where $X$ represents the position of the grey wolf, $X_{P}$ represents the position of the prey and $t$ presents the current iteration. The co-efficient vector parameters, $A$ and $C$, can be determined by:

$$
\begin{gathered}
A=2 a \cdot r_{1}-a \quad \& C=2 \cdot r_{2} \\
a=2-2\left(\frac{t}{T_{\max }}\right) \quad(11)
\end{gathered}
$$

where $a$ is a linearly decreasing component from 2 to $0, t$ is the current iteration, $T_{\max }$ is the maximum number of iterations, and $r_{1}$ and $r_{2}$ are two random values between 0 and 1 .

When the searching and tracking of the prey location is completed by the alpha, beta, and delta wolves, they lead the omega wolves to surround the prey. By assuming the prey location, the best three solutions are stored for updating the position of the other grey wolves in the group. The equation for updating the grey wolf position is:

$$
X(t+1)=\left(\frac{X_{1}+X_{2}+X_{3}}{3}\right)
$$

where each position of $X_{1}, X_{2}$, and $X_{3}$ can be updated as:

$$
\left\{\begin{array}{l}
X_{1}=\left|X_{\alpha}-A_{1} \cdot D_{\alpha}\right| \\
X_{2}=\left|X_{\beta}-A_{2} \cdot D_{\beta}\right| \\
X_{3}=\left|X_{\delta}-A_{3} \cdot D_{\delta}\right|
\end{array}\right.
$$

$$
\left\{\begin{array}{l}
D_{\alpha}=\left|C_{1} \cdot X_{\alpha}-X\right| \\
D_{\beta}=\left|C_{2} \cdot X_{\beta}-X\right| \\
D_{\delta}=\left|C_{3} \cdot X_{\delta}-X\right|
\end{array}\right.
$$

The binary conversion of the grey wolf technique can be obtained by the sigmoid conversion of the position vectors presented in [21]. The updated position of the grey wolves in the binary format can be represented by:.

$$
X(t+1)=\left\{\begin{array}{c}
1, \text { if } S F \geq r_{3} \\
0, \text { otherwise }
\end{array}\right.
$$

where $r_{3}$ is a random number in $[0,1]$ and $S F$ represents the sigmoid function and can be written as:

$$
S F=\frac{1}{1+\exp \left(-10\left(\frac{X_{1}+X_{2}+X_{3}}{3}-0.5\right)\right)}
$$

The flowchart of the proposed BGWO technique is presented in Figure 2 and the steps to find the optimal PMUs along with their placement locations are explained below:

Step 1: Initialize the total population of the grey wolves, the maximum number of iterations (max iter), and $A \& C$ parameters.

Step 2: Then randomly initialize the position of PMUs and calculate the objective function value for each alpha wolf solution.

Step 3: Update the three searching agents and compute $X_{\alpha}$, $X_{\beta}$, and $X_{\delta}$ using (13).

Step 4: Update the position of each wolf with (15) after converting it into binary form.

Step 5: Repeat step 2 to step 4 up to the max iter. Finally, the $X_{\alpha}$ gives the optimal PMU numbers along with its optimal positions.

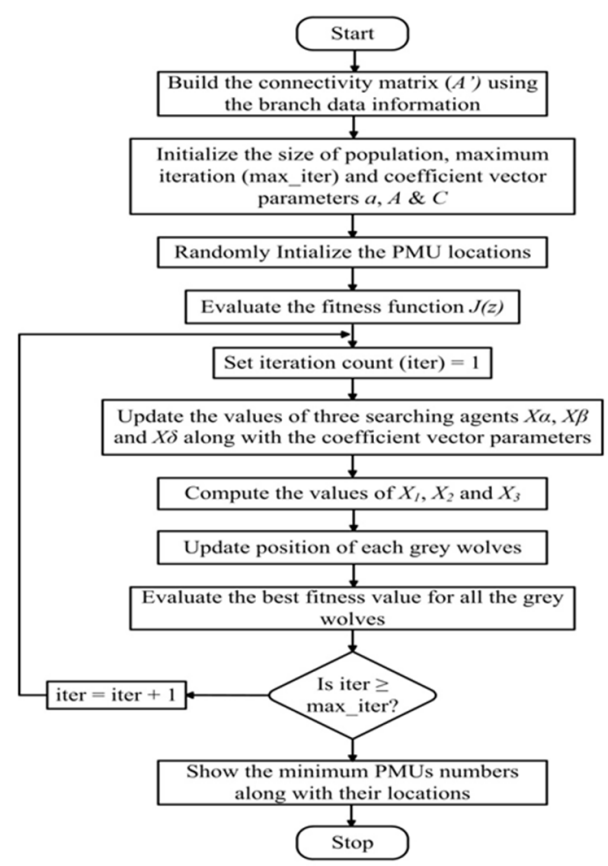

Fig. 2. Flowchart of the proposed BGWO for the OPP problem. 


\section{RESULTS AND DISCUSSION}

The proposed BGWO was implemented on several IEEE test node feeders including IEEE $-13,-33,-37$, and -123 node systems to solve the PMU placement problem using the MATLAB 2019 version. The proposed technique is simulated considering two conditions: Under normal operation and under a single PMU loss. In this paper the channel limit is considered to be fixed for the optimal placement of PMUs and it is based on the maximum branches connected to a node in the feeder network. Therefore the maximum channel limit is set to 5 for all the test feeders except the IEEE 33 node test feeder where the maximum channel is considered as 4 . The value of each parameter used in the simulation is given in Table I. Table II shows the minimum PMUs required for the solution of the OPP problem along with their locations and CSOI value. The results obtained under normal operating conditions satisfy the observability criterion. The results show that a total of 6 PMUs are required for the IEEE 13 system. The basic structure of the network is given in Figure 3.

TABLE I. VALUES OF CONSIDERED PARAMETERS

\begin{tabular}{|c|c|}
\hline Parameters & Values \\
\hline Population taken & 50 to 200 \\
\hline Maximum iteration number & 300 \\
\hline Weight value for the measurement redundancy $\left(w_{1}\right)[18]$ & 0.01 \\
\hline Weight value for the number of observable nodes $\left(w_{2}\right)[18]$ & -2 \\
\hline
\end{tabular}

TABLE II. RESULTS OF THE OPP PROBLEM WITH CSOI VALUE UNDER NORMAL OPERATION

\begin{tabular}{|c|c|c|c|}
\hline $\begin{array}{c}\text { Test } \\
\text { node } \\
\text { feeders }\end{array}$ & PMU locations & CSOI & $\begin{array}{c}\text { Optimal } \\
\text { number } \\
\text { of } \\
\text { PMUs }\end{array}$ \\
\hline \multirow{5}{*}{ IEEE-13 } & $3,4,5,8,9,10$ & 23 & \multirow{5}{*}{6} \\
\hline & $3,4,6,8,9,10$ & 22 & \\
\hline & $2,4,5,8,9,10$ & 22 & \\
\hline & $2,4,5,8,9,11$ & 21 & \\
\hline & $2,4,6,8,9,11$ & 20 & \\
\hline \multirow{5}{*}{ IEEE-33 } & $2,4,8,11,14,17,21,24,26,29,32$ & 34 & \multirow{5}{*}{11} \\
\hline & $2,5,8,11,14,17,21,24,27,29,32$ & 34 & \\
\hline & $2,5,8,11,14,17,21,24,27,30,32$ & 34 & \\
\hline & $2,5,8,11,14,17,21,24,26,29,32$ & 34 & \\
\hline & $2,5,8,11,14,17,21,24,27,30,33$ & 33 & \\
\hline \multirow{4}{*}{ IEEE-37 } & $2,3,6,9,12,15,19,22,28,30,33,35$ & 47 & \multirow{4}{*}{12} \\
\hline & $1,3,6,9,12,15,19,22,28,30,33,35$ & 46 & \\
\hline & $2,3,6,9,12,15,19,22,29,30,33,35$ & 46 & \\
\hline & $1,3,6,9,12,15,19,22,29,30,33,35$ & 45 & \\
\hline \multirow{2}{*}{$\begin{array}{c}\text { IEEE- } \\
123\end{array}$} & $\begin{array}{c}1,3,5,8,14,15,19,21,23,25,27,30,31,36 \\
38,40,42,45,47,51,53,55,58,60,63,65,67 \\
, 70,74,76,78,82,84,87,89,91,93,95,97 \\
100,103,106,108,110,113\end{array}$ & 162 & \multirow{2}{*}{45} \\
\hline & $\begin{array}{c}1,3,5,8,14,15,19,21,23,25,27,30,31,36 \\
38,40,42,45,47,51,52,55,58,60,63,65,67 \\
, 70,72,74,78,82,84,87,89,91,93,95,97, \\
100,103,106,108,110,113\end{array}$ & 162 & \\
\hline
\end{tabular}

For the IEEE 33 test node feeder as shown in Figure 4, 4 sets of placement locations have been obtained with a maximum CSOI of 34 by reducing the number of PMUs to 11 . Similarly, 12 PMUs with a maximum of 47 CSOI have been obtained for the IEEE 37 node system (Figure 5). To verify the efficiency, a large size IEEE 123 test node feeder network has also been considered. The IEEE 123 test feeder has 123 nodes containing several switches. Here, there are 119 nodes in the network and the basic topology is given in Figure 6. The proposed technique has given 45 PMUs obtained with a maximum CSOI limit of 162 by solving the OPP problem. The convergence graphs of all the four test feeders are shown in Figure 7 over a maximum of 300 iterations.

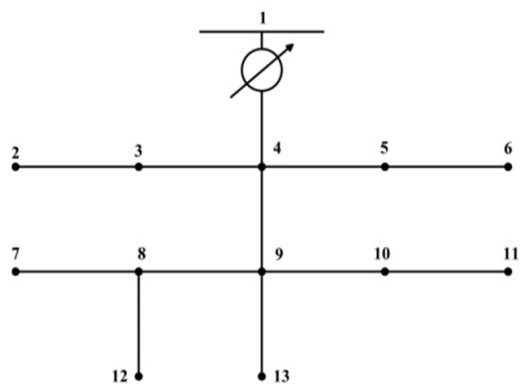

Fig. 3. Schematic diagram of the IEEE 13 node test feeder.

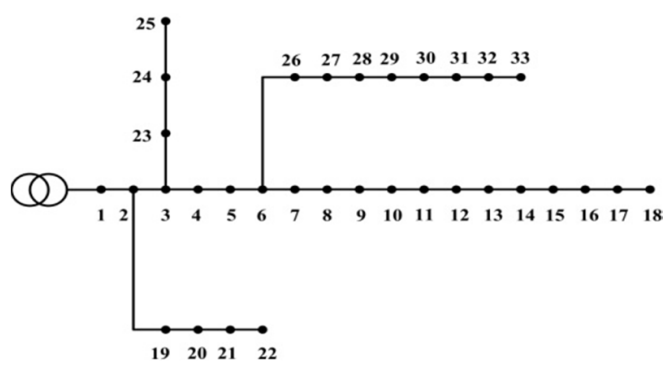

Fig. 4. Schematic diagram of the IEEE 33 node test feeder.

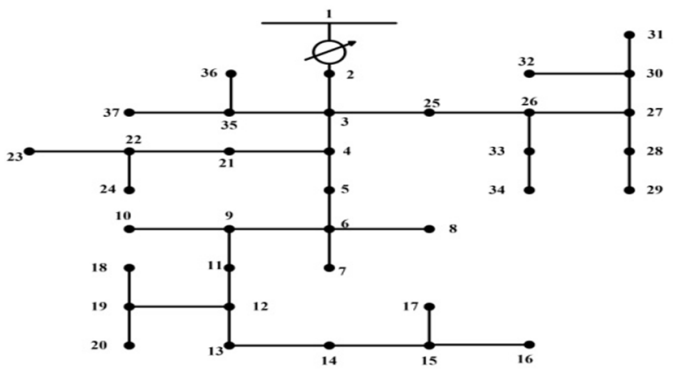

Fig. 5. Schematic diagram of the IEEE 37 node test feeder.

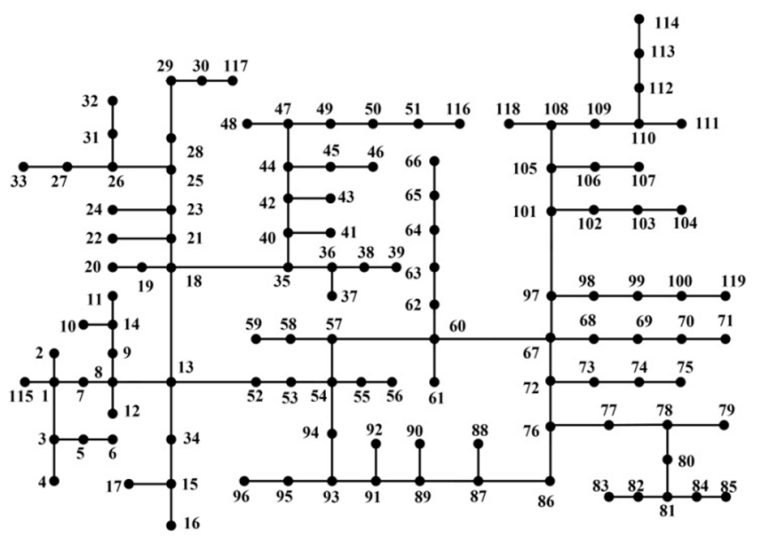

Fig. 6. Schematic diagram of the IEEE 123 node test feeder. 


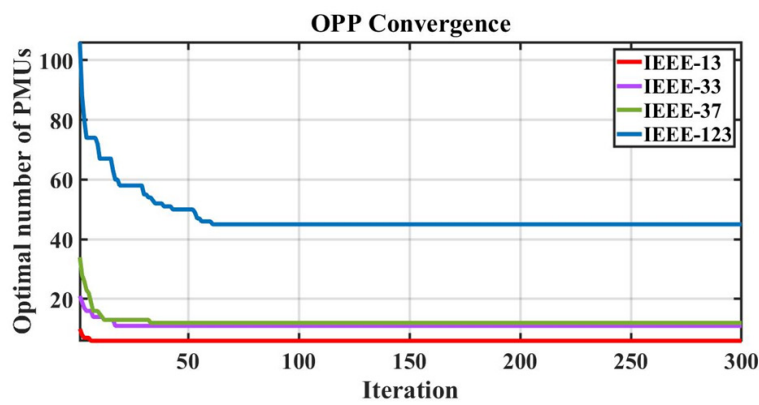

Fig. 7. Convergence graph of all the IEEE test node feeders under normal condition.

The OPP solutions under a single PMU loss are given in Table III. It can be observed that the numbers of PMUs in this case are increasing twice than the normal operating condition except for the IEEE 13 node system. The condition to maintain complete observability during the single PMU loss is to observe each node by two PMUs. Therefore, in order to ensure that the feeder networks remain observable, the obtained results are quite larger than the normal condition. For the IEEE-13 node test feeder, the number of PMUs obtained is the same as the total number of nodes in the system. Any further reduction in the obtained solution fails to satisfy the full observability criterion. Therefore it is not included in the tabulation. But except the IEEE-13 system all other feeder networks have given smaller numbers of PMUs.

TABLE III. RESULTS OF THE OPP PROBLEM WITH CSOI VALUES UNDER A SINGLE PMU LOSS

\begin{tabular}{|c|c|c|}
\hline Test node feeders & Optimal number of PMUs & CSOI \\
\hline IEEE-33 & 24 & 70 \\
\hline IEEE-37 & 31 & 90 \\
\hline IEEE-123 & 97 & 282 \\
\hline
\end{tabular}

A comparative analysis of the OPP solution between the proposed approach and previously developed methods is presented in Table IV. It has been observed that only the conventional method is implemented to solve the placement problem for the distribution network. As these methods have the disadvantage of finding local minima for large networks, it is therefore advised to opt for heuristic methods over conventional methods. From the comparison, it has been observed that the number of PMUs obtained from the proposed method is similar, but the CSOI values are different. The results have either equal or higher CSOI value. The computational time obtained from each feeder network is given in Table $\mathrm{V}$ and the convergence plots are shown in Figure 8.

TABLE IV. COMPARATIVE RESULTS UNDER NORMAL OPERATION

\begin{tabular}{|c|c|c|c|c|}
\hline \multirow{2}{*}{ Methods } & \multicolumn{4}{|c|}{ Test Feeders with their CSOI } \\
\cline { 2 - 5 } & IEEE-13 & IEEE-33 & IEEE-37 & IEEE-123 \\
\hline Proposed & $6(23)$ & $11(34)$ & $12(47)$ & $45(162)$ \\
\hline Binary ILP [3] & - & $11(\mathrm{NA})$ & - & - \\
\hline Binary ILP [4] & - & $11(\mathrm{NA})$ & - & $45(\mathrm{NA})$ \\
\hline Hybrid ILP [5] & - & $11(34)$ & - & $45(162)$ \\
\hline Linear programming [22] & $6(16)$ & - & $12(43)$ & $48(148)$ \\
\hline ILP [23] & - & $17(\mathrm{NA})$ & - & - \\
\hline
\end{tabular}

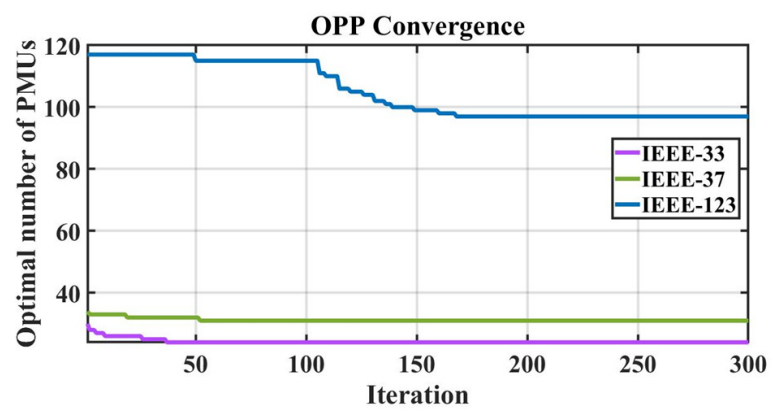

Fig. 8. Convergence graphs for the IEEE-33, -37, and -123 feeder networks under a single PMU loss condition.

TABLE V. COMPUTATIONAL TIME

\begin{tabular}{|c|c|c|}
\hline Test node feeders & $\begin{array}{c}\text { Normal operating } \\
\text { condition (s) }\end{array}$ & $\begin{array}{c}\text { Single PMU loss } \\
\text { (s) }\end{array}$ \\
\hline IEEE-13 & 1.76 & - \\
\hline 1EEE-33 & 2.23 & 2.31 \\
\hline IEEE-37 & 2.38 & 2.55 \\
\hline IEEE-123 & 27.42 & 27.71 \\
\hline
\end{tabular}

These solutions are consistent when compared with the prior studies. Hence, it has been proved that the proposed metaheuristic based technique is capable to solve the PMU placement problem while achieving full observability with maximum measurement redundancy for the distribution network.

\section{CONCLUSION}

A binary GWO technique has been proposed in this work to solve the OPP problem in the distribution network by finding a reduced number of PMUs along with their placement locations to achieve complete system observability. The maximum redundancy concept has been considered as another important parameter in the objective function for the best PMU placement location among several possible sets. Four different IEEE test node feeders have been taken into consideration for the validation of the proposed technique during the normal operating and the single PMU loss conditions. The results obtained from the proposed method indicate that this technique is capable to reduce the PMUs with maximum measurement redundancy to the other approaches. The proposed BGWO is simple and easy to implement in both transmission and distribution networks.

\section{REFERENCES}

[1] A. G. Phadke, J. S. Thorp, and K. J. Karimi, "State Estimlatjon with Phasor Measurements," IEEE Transactions on Power Systems, vol. 1, no. 1, pp. 233-238, Feb. 1986, https://doi.org/10.1109/TPWRS.1986. 4334878.

[2] N. V. P. Babu, P. S. Babu, and D. V. S. S. S. Sarma, "A New Power System Restoration Technique based on WAMS Partitioning," Engineering, Technology \& Applied Science Research, vol. 7, no. 4, pp. 1811-1819, Aug. 2017, https://doi.org/10.48084/etasr.1197.

[3] H. Su, C. Wang, P. Li, Z. Liu, L. Yu, and J. Wu, "Optimal placement of phasor measurement unit in distribution networks considering the changes in topology," Applied Energy, vol. 250, pp. 313-322, Sep. 2019, https://doi.org/10.1016/j.apenergy.2019.05.054.

[4] Z. Zhao et al., "Optimal placement of PMUs and communication links for distributed state estimation in distribution networks," Applied 
Energy, vol. 256, p. 113963, Dec. 2019, https://doi.org/10.1016/ j.apenergy.2019.113963.

[5] X. Chen et al., "Full Coverage of Optimal Phasor Measurement Unit Placement Solutions in Distribution Systems Using Integer Linear Programming," Energies, vol. 12, no. 8, p. 1552, Jan. 2019, https://doi.org/10.3390/en12081552.

[6] A. Ahmadi, Y. Alinejad-Beromi, and M. Moradi, "Optimal PMU placement for power system observability using binary particle swarm optimization and considering measurement redundancy," Expert Systems with Applications, vol. 38, no. 6, pp. 7263-7269, Jun. 2011, https://doi.org/10.1016/j.eswa.2010.12.025.

[7] A. A. Saleh, A. S. Adail, and A. A. Wadoud, "Optimal phasor measurement units placement for full observability of power system using improved particle swarm optimisation," Transmission Distribution IET Generation, vol. 11, no. 7, pp. 1794-1800, 2017, https://doi.org/ 10.1049/iet-gtd.2016.1636.

[8] H. H. Müller and C. A. Castro, "Genetic algorithm-based phasor measurement unit placement method considering observability and security criteria," Transmission Distribution IET Generation, vol. 10, no. 1, pp. 270-280, 2016, https://doi.org/10.1049/iet-gtd.2015.1005.

[9] B. Milosevic and M. Begovic, "Nondominated sorting genetic algorithm for optimal phasor measurement placement," IEEE Transactions on Power Systems, vol. 18, no. 1, pp. 69-75, Feb. 2003, https://doi.org/ 10.1109/TPWRS.2002.807064.

[10] M. T. Mouwafi, R. A. El-Sehiemy, A. A. Abou El-Ela, and A. M. Kinawy, "Optimal placement of phasor measurement units with minimum availability of measuring channels in smart power systems," Electric Power Systems Research, vol. 141, pp. 421-431, Dec. 2016, https://doi.org/10.1016/j.epsr.2016.07.029.

[11] S. Li and Z. Meng, "Optimal PMU placement based on improved binary artificial bee colony algorithm," in 2017 IEEE Transportation Electrification Conference and Expo, Asia-Pacific (ITEC Asia-Pacific), Harbin, China, Aug. 2017, pp. 1-6, https://doi.org/10.1109/ITECAP.2017.8081008.

[12] S. P. Singh and S. P. Singh, "A Multi-objective PMU Placement Method in Power System via Binary Gravitational Search Algorithm," Electric Power Components and Systems, vol. 45, no. 16, pp. 1832-1845, Oct. 2017, https://doi.org/10.1080/15325008.2017.1378775.

[13] T. Prakash, V. P. Singh, S. Singh, and S. Mohanty, "Binary Jaya algorithm based optimal placement of phasor measurement units for power system observability," Energy Conversion and Management, vol. 140, pp. 34-35, 2017.

[14] N. C. Koutsoukis, N. M. Manousakis, P. S. Georgilakis, and G. N. Korres, "Numerical observability method for optimal phasor measurement units placement using recursive Tabu search method," IET Generation, Transmission \& Distribution, vol. 7, no. 4, pp. 347-356, Apr. 2013, https://doi.org/10.1049/iet-gtd.2012.0377.

[15] A. Raj and C. Venkaiah, "Optimal PMU placement by teaching-learning based optimization algorithm," in 2015 39th National Systems Conference (NSC), Noida, India, Dec. 2015, https://doi.org/10.1109/NATSYS.2015.7489080.

[16] S. Priyadarshini and C. K. Panigrahi, "Binary Grey Wolf technique for Optimal Placement of Phasor Measurement Unit with full network observability," Journal of Engineering Science and Technology, vol. 15, no. 5, pp. 2924-2938, Oct. 2020.

[17] N. H. A. Rahman, A. F. Zobaa, and M. Theodoridis, "Improved BPSO for optimal PMU placement," in 2015 50th International Universities Power Engineering Conference (UPEC), Stoke on Trent, UK, Sep. 2015, pp. 1-4, https://doi.org/10.1109/UPEC.2015.7339885.

[18] N. H. A. Rahman and A. F. Zobaa, "Integrated Mutation Strategy With Modified Binary PSO Algorithm for Optimal PMUs Placement," IEEE Transactions on Industrial Informatics, vol. 13, no. 6, pp. 3124-3133, Dec. 2017, https://doi.org/10.1109/TII.2017.2708724.

[19] R. Manam and S. R. Rayapudi, "Sensitive Constrained Optimal PMU Allocation with Complete Observability for State Estimation Solution," Engineering, Technology \& Applied Science Research, vol. 7, no. 6, pp. 2240-2250, Dec. 2017, https://doi.org/10.48084/etasr.1542.
[20] S. Mirjalili, S. M. Mirjalili, and A. Lewis, "Grey Wolf Optimizer," Advances in Engineering Software, vol. 69, pp. 46-61, Mar. 2014, https://doi.org/10.1016/j.advengsoft.2013.12.007.

[21] J. Too, A. R. Abdullah, N. Mohd Saad, N. Mohd Ali, and W. Tee, "A New Competitive Binary Grey Wolf Optimizer to Solve the Feature Selection Problem in EMG Signals Classification," Computers, vol. 7, no. 4 , p. 58 , Dec. 2018 , https://doi.org/10.3390/computers7040058.

[22] M. Spitzer, C. Eerdmans, A. S. Nair, and P. Ranganathan, "Evaluation of PMU Placements with SORI and ORC Indices for IEEE Test Feeders," in 2018 IEEE International Conference on Electro/Information Technology (EIT), Rochester, MI, USA, May 2018, pp. 0687-0690, https://doi.org/10.1109/EIT.2018.8500263.

[23] V. Tran, H. Zhang, and V. Nguyen, "Optimal PMU Placement in Multiconfiguration Power Distribution Networks," in Proceedings of the 2016 4th International Conference on Electrical \& Electronics Engineering and Computer Science (ICEEECS 2016), Dec. 2016, pp. 508-514, https://doi.org/10.2991/iceeecs-16.2016.104. 